\title{
Histopathological pattern of central nervous system tumor: A three year retrospective study
}

\author{
Aryal $\mathrm{G}^{1}$
}

${ }^{1}$ Department of Pathology, KIST Medical College and Teaching Hospital, Kathmandu, Nepal

\author{
Keywords: \\ Astrocytoma; \\ Meningioma; \\ Tumor; \\ Nepal; \\ Central nervous system
}

\begin{abstract}
Background: There is no documented data regarding the histopathological spectrum of the central nervous system (CNS) tumors in Nepal. The aim of this study was to establish the relative frequency of biopsy proven tumors of the CNS from a single university hospital in Nepal.

Materials and Methods: In the period between1998-2000, we retrospectively analyzed data on 57 patients (28 male and 29 female) diagnosed with CNS tumors according to the World Health Organization's diagnostic criteria. Patient data were retrieved from the archives of the department of Pathology, Nepal Medical College.

Results: A total of 57 CNS tumors were diagnosed during a three year period. Of these, $49(86 \%)$ were primary, and $8(14 \%)$ were metastatic. The most frequent type of CNS tumors was astrocytoma (22 cases, $38.6 \%$ ), followed by meningioma ( 8 cases, $14 \%$ ) and metastatic tumor ( 8 cases, $14 \%)$. Among the 8 metastatic tumors, the most common histologic type was adenocarcinoma ( 7 cases, $87.5 \%$ ).

Conclusion: The most frequent type of CNS tumors in this study was astrocytoma, followed by meningioma and metastatic tumor. The ratio of male to female for all CNS tumors was 0.9:1. Female outnumbered male in meningioma (1:0.3).
\end{abstract}

\section{INTRODUCTION}

The central nervous system (CNS) tumors that predominate in adults differ from those seen in children. Most pediatric neoplasms occur in the posterior fossa. In adults, the majority of CNS tumors arise in the anterior fossa.

The glial tumors include astrocytoma, ependymoma, glioblastoma, oligodendroglioma and various subtypes. It

\section{Correspondence:}

Dr. Gopi Aryal, MD, PhD

Department of Pathology, KIST Medical College and Teaching Hospital GPO Box: 11442, Kathmandu, Nepal

E-mail: gopiaryal@hotmail.com is important to identify the oligodendroglial component in order to determine the most effective chemotherapy to use for these gliomas. Non-glial tumors includes embryonal tumors, choroid plexus tumors, pineal tumors, meningeal tumors, germ cell tumors, tumors of the sellar region and tumors of hematopoietic cell origin.

The fourth edition of the World Health Organization (WHO) classification of tumors of the CNS, published in 2007, lists several new entities, including angiocentric glioma, papillary glioneuronal tumor, rosette-forming glioneuronal tumor of the fourth ventricle, papillary tumor of the pineal region, pituicytoma and spindle cell oncocytoma of the adenohypophysis. ${ }^{1}$ The exact histological diagnosis of 
CNS tumors is essential to predict the prognostic factors. The new diagnostic criteria and techniques have affected the relative frequencies of CNS tumors. There have been no statistical reports on the CNS tumors in Nepal. In this study, the relative frequency of CNS tumors, including tumors of the cranial and paraspinal nerves, have been determined by analyzing 57 cases according to the revised WHO classification.

\section{MATERIALS AND METHODS}

A total of 57 cases of CNS tumors were retrieved from the archives of the department of Pathology, Nepal Medical College from January 1998 to December 2000. The diagnoses in all the cases were made on histopathological examination of routinely processed tissue. The Hematoxylin and Eosin stained sections in all cases were reviewed by the authors and diagnosis was confirmed applying revised WHO classification. The relative frequency of tumors and the distribution of age, sex and location were analyzed. The statistical analysis was done by using Statistical Package for Social Science (SPSS) version 17 for windows.

\section{RESULTS}

A total of 57 CNS tumors were diagnosed during three year period. Of these, $49(86 \%)$ were primary, and $8(14 \%)$ were metastatic. The most frequent type of CNS tumor was astrocytoma (22 cases, $38.6 \%$ ), located in the frontoparietal cortex, followed by meningioma ( 8 cases, 14\%) and metastatic tumor ( 8 cases, 14\%). Of the 8 metastatic tumors, the most common histological type was adenocarcinoma ( 7 cases, $87.5 \%$ ).

Table 1: The relative frequencies of CNS tumors

\begin{tabular}{lcc}
\hline Histological type & No. of cases & \% of total cases \\
\hline Astrocytoma & 22 & 38.6 \\
Meningioma & 8 & 14.0 \\
Metastatic tumor & 8 & 14.0 \\
Pituitary adenoma & 3 & 5.2 \\
Neurilemmoma & 3 & 5.2 \\
Medulloblastoma & 2 & 3.5 \\
Craniopharyngioma & 2 & 3.5 \\
PNET* & 2 & 3.5 \\
Neurofibroma & 2 & 3.5 \\
Gliomatosis cerebri & 1 & 1.8 \\
Ependymoma & 1 & 1.8 \\
Hemangioma & 1 & 1.8 \\
Hemangiopericy- & 1 & 1.8 \\
toma & 1 & 1.8 \\
Lymphoma & $\mathbf{5 7}$ & $\mathbf{1 0 0 . 0}$ \\
\hline Total & &
\end{tabular}

* PNET (Supratentorial primitive neuroectodermal tumor).
The relative frequency of CNS tumors is given in Table 1.

\section{Tumor of neuroepithelial tissue}

Among the 28 tumors of neuroepithelial tissue, the astrocytic tumors were the most common histologic type ( 22 cases, $78.6 \%$ ) followed by medulloblastoma ( 2 cases, $7.1 \%$ ), supratentorial primitive neuroectodermal tumors (PNET, 2 cases, 7.1\%), ependymoma (1 case, 3.6\%) and gliomatosis cerebri (1 case, $3.6 \%)$. The most common type of astrocytoma was WHO grade IV type (Table 2).

\section{Tumor of non-glial tissue}

Of the 8 cases of meningeal tumor, there was only 1 case of atypical meningioma, WHO grade II $(12.5 \%)$. The remaining meningiomas were $\mathrm{WHO}$ grade I with low risk of recurrence and aggressive growth. The nerve sheath tumors consisted of neurilemmoma (3 cases, 5.2\%) and neurofibroma ( 2 cases, $3.5 \%$ ). The cases of pituitary adenoma were positive for pituitary hormones. Among hematopoietic tumors, there was 1 case of CNS lymphoma and 1 case of cavernous hemangioma. Both cases of craniopharyngioma were adamantiomatous type consisting of broad strands, cord and bridges of a multistratified squamous epithelium with peripheral palisading of nuclei.

\section{Metastatic tumor}

Among the 8 metastatic tumors, the most common histologic type was adenocarcinoma ( 7 cases, $87.5 \%$ ). There was 1 case of hepatocellular carcinoma (12.5\%).

Table 2: Relative frequency of astrocytoma according to WHO grading

\begin{tabular}{lcc}
\hline WHO grading* & No. of cases & \% of total cases \\
\hline Grade I & 6 & 27.3 \\
Grade II & 6 & 27.3 \\
Grade III & 3 & 13.6 \\
Grade IV & 7 & 31.8 \\
\hline Total & $\mathbf{2 2}$ & $\mathbf{1 0 0 . 0}$ \\
\hline${ }^{*}$ Reference I & & \\
\end{tabular}

Table 3: Age and sex distribution of CNS tumors

\begin{tabular}{lcc}
\hline Histological type & Peak age group in years & $\begin{array}{c}\text { Gender ratio } \\
\text { (M:F) }\end{array}$ \\
\hline Astrocytoma & $21-40$ & $1.2: 1$ \\
Meningioma & $21-40$ & $0.3: 1$ \\
Metastatic tumor & $41-60$ & $0.4: 1$ \\
Pituitary adenoma & $21-40$ & $2: 1$ \\
\hline
\end{tabular}




\section{Age and Sex Distribution}

The age and sex distribution of four major types of CNS tumors are tabulated in Table 3. The ratio of male to female for all CNS tumors was 0.9:1. Female outnumbered male in meningioma (1:0.3). Astrocytoma, meningioma and pituitary adenomas showed predilection for those in their twenties to forties. The youngest patient for medulloblastoma and craniopharyngioma were 9 years and 5 years of age, respectively. Metastatic tumors occur frequently between 40 and 60 years of age. The most common sites for astrocytoma and meningioma were the frontoparietal region (12 cases, $54.5 \%$ ) and the thalamus ( 6 cases, $75 \%$ ) respectively.

\section{DISCUSSION}

In the present study we observed that the CNS tumor that had the highest incidence was astrocytoma (38.6\%).

There have been several important reviews of CNS tumors regarding the incidence and relative percentages of these neoplasms in the United States, Europe and Asia. The most common CNS tumors were astrocytoma in United States $(49.6 \%)^{2}$, Taiwan $(32.9 \%)^{3}$, Mexico $(33 \%)^{4}$ and Germany $(41.7 \%))^{5}$

In a similar study from Croatia, the most frequent intracranial tumors were those of neuroepithelial tissue $(58.3 \%){ }^{6}$

Histological grading is a means of predicting the biological behavior of neoplasm. The grading factors influence the choice of therapy. The most common type of astrocytoma in our study was WHO grade IV type. The late presentation of our patients to the hospital could be the possible cause.

In contrast to our study, the most common primary CNS tumors in Korea was meningioma (31.2\%) followed by glioblastoma (30.7\%). ${ }^{7}$ Meningioma was the most common tumor among clinically diagnosed tumors, followed by neuroepithelial tumor, schwannoma, and pituitary tumor among atomic bomb survivors in Hiroshima and Nagasaki, Japan. ${ }^{8}$ The difference in the relative frequency and the tumor distribution among populations in different countries may be due to genetic and environmental factors. In a report from USA, meningiomas were the only tumors with a significant excess in females. ${ }^{9}$

The second most common type of primary CNS tumor in our study was meningioma (14\%), followed by pituitary adenoma (5.2\%) and neurilemmoma (5.2\%). There were also 2 cases of medulloblastoma $(3.5 \%)$ and 2 cases of craniopharyngioma (3.5\%). The age group of the cases of medulloblastoma and craniopharyngioma ranged from 5 to 37 years, though they were commonly reported in children. ${ }^{10}$

Among the 8 metastatic tumors, the most common histological type was adenocarcinoma ( 7 cases, $87.5 \%$ ). The common age group was 41-60 years of age. The findings are comparable to a study done in Korea. ${ }^{11}$

The ratio of male to female for all CNS tumors in our study was 0.9:1 in contrast to study from Puerto Rico where CNS tumors were more common in females. ${ }^{12}$

It is difficult to compare these different studies due to the lack of uniformity in the case material, along with differences in the study methodologies. This study may not represent an accurate incidence of CNS tumors in Nepal due to the limited number of cases. Furthermore, the study was based on a single center analysis.

\section{CONCLUSION}

The most frequent type of CNS tumors in this study was astrocytoma, followed by meningioma and metastatic tumor. There is no documented data regarding the incidence of CNS tumors in Nepal. This study may provide the representative incidence of various types of CNS tumors. A nationwide multicenter study is necessary in the future.

\section{AKNOWLEDGEMENT}

I would like to thank Mr. Ram Thapa, department of pathology, Nepal Medical College, for extracting data from the hospital database.

\section{REFERENCES}

1. Louis DN, Ohgaki H, Wiestler OD et al. The 2007 WHO classification of tumours of the central nervous system. Acta Neuropathol 2007;114:97-109.

2. Walker AE, Robin M, Weinfeld FD. Epidemiology of brain tumors: the national survey of intracranial neoplasm. Neurol 1985;35:219-26.

3. Kepes JJ, Chen WY, Pang LC, Kepes M. Tumor of central nervous system in Taiwan, Republic of China. Surg Neurol 1984;22:149-56.

4. Lopez Gonzalez MA, Sotelo Z. Brain tumors in Mexico: characteristics and prognosis of glioblastoma. Surg Neurol 2000;53:157-62.

5. Kaatsch P, Rickert CH, Kühl J, Schüz J, Michaelis J. Populationbased epidemiologic data on brain tumors in German children. Cancer 2001;92:3155-64.

6. Materljan E, Materljan B, Sepcić J, Tuskan-Mohar L, Zamolo G, Erman-Baldini I. Epidemiology of central nervous system tumors in Labin area, Croatia, 1974-2001. Croat Med J 2004;45:206-12.

7. Lee CH, Jung KW, Yoo H, Park S, Lee SH. Epidemiology of primary brain and central nervous system tumors in Korea. J Korean Neurosurg Soc 2010;48:145-52.

8. Yonehara S, Brenner AV, Kishikawa $\mathrm{M}$ et al. Clinical and epidemiologic characteristics of first primary tumors of the central nervous system and related organs among atomic bomb survivors in Hiroshima and Nagasaki, 1958-1995. Cancer 2004;101:1644-54.

9. Surawicz TS, McCarthy BJ, Kupelian V, Jukich PJ, Bruner JM, Davis FG. Descriptive epidemiology of primary brain and CNS tumors: results from the , Central Brain Tumor Registry of the United States, 1990-1994. Neuro Oncol 1999;1:14-25.

10. Kuratsu J, Takeshima H, Ushio Y. Trends in the incidence of primary intracranial tumors in Kumamoto, Japan. Int J Clin Oncol 2001;6:183-91. 
11. Suh YL, Koo H, Kim TS, Chi JG et al. Tumors of the central nervous system in Korea: a multicenter study of 3221 cases. Neurooncol 2002;56:251-9.
12. Murray G, Jiménez L, Báez F, Colón-Castillo LE, Brau RH. Descriptive profile of surgically-confirmed adult central nervous system tumors in Puerto Rico. P R Health Sci J 2009; 28: 317-28. 